\title{
New perspectives on COVID-19 and pregnancy
}

\author{
Ana Gabriela Turcu Duminica', Viorel Alexandrescu' ${ }^{2}$, Anca Marina Ciobanu', \\ Corina Gica', Radu Botezatu ${ }^{1,3}$, Nicolae Gica ${ }^{1,3}$, Gheorghe Peltecu ${ }^{1,3}$, \\ Anca Maria Panaitescu ${ }^{1,3}$ \\ ${ }^{1}$ Filantropia Clinical Hospital, Bucharest, Romania \\ 2"Ion Cantacuzino" National Institute for Medical Military Research Development, \\ Bucharest, Romania \\ 3"Carol Davila" University of Medicine and Pharmacy, Bucharest, Romania
}

\begin{abstract}
Although there have been various pandemics throughout the history of humankind, modern age was thought to be ready to face such a challenge by implementing an advanced health care system. However, epidemic outbreaks are ubiquitous, and they have a substantial potential to evolve into pandemics, hence this being the actual context of SARS-CoV-2 pandemics from 2019. The difficulty of the addressed subject consists in the fact that the majority of pharmacological clinical studies omited pregnant patients. Acknowledging the physiological changes, pregnant women represent an unique group of patients as pregnancy could embody a particular substrate for this contemporary type of infection.
\end{abstract}

Keywords: pregnancy, COVID-19, SARS-CoV-2, diagnosis, treatment

\section{INTRODUCTION}

The first case of infection with the new coronavirus SARS-CoV-2 was reported in the city of Wuhan, China, in December 2019 and a few months later, in March 2020, the World Health Organization (WHO) officially acknowledged it as pandemic. Recent studies have shown that respiratory infections, including those acquired from coronaviruses such as Severe Acute Respiratory Syndrome (SARS) and Middle East Respiratory Syndrome (MERS) act like comorbidities in pregnancy, having a meaningful role, not only in the rate of preterm birth, but also in miscarriage and preeclampsia [1]. As pregnancy is characterized by a particular state in which infections and complications alter its physiological development, it is premature to anticipate the impact of the medication used in SARS-CoV-2 infection in pregnant women.

\section{OBJECTIVE AND METHOD}

In the present systematic review, we aimed to revise the main symptoms and vertical transmission of SARS-CoV-2 in pregnant patients. We added few comments on pregnancy outcomes and influence of seasonal vaccine on COVID-19 patients. Literature was searched via Pubmed using keywords such as pregnancy, COVID-19, SARS-CoV-2, diagnosis, treatment, with 11 papers included in the final review.

\section{SARS-COV-2 INFECTION ONSET SYMPTOMS}

Onset symptoms remain the first steps in recognizing the SARS-CoV-2 infection, although they vary from the lack of symptoms to gastrointestinal manifestations, as shown in Table 1 [1]. It is noted that most patients presented with high temperature 
and cough, progressing into breathing complications, as well as lethargy, body weakness or gastrointestinal manifestations.

TABLE 1. Common debut symptoms of SARS-CoV-2 infection [1]

\begin{tabular}{|l|}
\hline Lack of symptoms \\
\hline High temperature \\
\hline Alteration of taste or smell sensation \\
\hline Muscle, limb, joint distress \\
\hline Lethargy, body weakness \\
\hline Cephalalgia \\
\hline Breathing complications \\
\hline Cough \\
\hline Diarrhea \\
\hline
\end{tabular}

\section{MATERNAL-FETAL TRANSMISSION OF SARS-COV-2 INFECTION}

An important topic that concerns obstetricians all around the world is the maternal fetal transmission of SARS-CoV-2 infection. In a systematic review of treatment and outcomes of pregnant women with COVID-19, Pastick KA et al. noted that, in numerous cases, SARS-CoV-2 was not identified in amniotic cavity/fluid, maternal milk, umbilical cord blood, placenta, vaginal secretions, as shown in Table 2 [2]. The same results were found in neonates, without proper identification of the new virus in neonatal serum or plasma, blood, feces, or gastric secretions [2].

TABLE 2. Pathway of maternal-fetal transmission of SARS-CoV-2 infection [2]

\begin{tabular}{|l|}
\hline Maternal \\
Amniotic cavity/fluid \\
Maternal milk \\
Placenta \\
Umbilical cord blood \\
Vaginal secretions \\
\hline Neonatal \\
Blood \\
Feces \\
Gastric secretions \\
Plasma \\
Serum
\end{tabular}

\section{PREGNANCY OUTCOMES}

In a study conducted by Dustin et al. in two maternity hospitals in Philadelphia, in a two-month period of time, $6.2 \%$ of pregnant patients were exposed to SARS-CoV-2, while the infection rate in the general population of Philadelphia was $1.4 \%$ at the same time [3]. Nonetheless, supplementary research is essential as pregnant patients have particular physiological characteristics in comparison to general population. Through the help of COVID-net, a general public accessible specific network hub that collects data with reference to SARS-CoV-2 patients, data available from 13 states was analyzed, concluding that fifty percent of SARS-CoV-2 parturient women admitted to hospital were asymptomatic [4]. Compared to general population, the rate of preterm births was larger in the SARS-CoV-2 group (12.6\% vs. 10\%) and three times greater in symptomatic women. Furthermore, higher rates of hospitalization were recorded in specific ethnic groups such as hispanic (42.5\% vs $15.3 \%)$ and black (26.5\% vs $19.5 \%)$ [4].

Although the first studies from China showed that pregnant women confirmed with SARS-CoV-2 infection have a higher risk of premature delivery, three recent studies [5,6,7] clearly showed the absence of an increased prevalence of preterm birth. The same lack of discrepancies were noted in Apgar scores and birth weight for gestational age between the group positive and negative for this viral infection [5].

These conflicting results show that we need more data to clarify this important aspect of premature delivery related to SARS-CoV-2 infection.

Pregnant women admitted in labor and tested positive for SARS-CoV-2 on admission are more likely to develop preeclampsia comparing to those tested negative $(7,7 \%$ vs. $4,3 \%)$ [7]. It is believed that even if COVID-19 is primarily a respiratory infection it also has systemic effects that may resemble to preeclampsia [8].

If one compares other variables referring to the maternal outcomes like preterm birth, way of delivery, postpartum hemorrhage, they did not significantly differ between groups [7].

\section{EPIDEMIOLOGIC ASPECTS OF SARS-COV-2 IN NEONATES}

Obstetrical health care providers are interested in the intrauterine transmission of SARS-CoV-2, albeit most recent studies have shown that if the mother is viremic during pregnancy, vertical transmission is possible and current recommendations are to quarantine a COVID-19 positive mother from the newborn [9].

Intrauterine infection has been documented but is uncommon. A recent study showed that in 155 neonates that were tested immediately after delivery, by RT-PCR from throat, all, except three cases were negative for SARS-CoV-2 [10]. 
Data from countries like China, Italy or Spain, that were severely affected by the SARS-CoV-2 pandemic, revealed that infant population does not represent a large category of patients with the characteristics of SARS-CoV-2 infection and with a prevalence of $50 \%$, the most common symptoms described were fever and non-productive cough [9].

\section{INFLUENCE OF SEASONAL VACCINE ON COVID-19 PATIENTS}

Respiratory infections caused by seasonal influenza, Streptococcus pneumoniae, measles, Bordetella pertussis and Haemophilus influenzae type b (Hib) could be prevented through immunization policies such as vaccination, with a meaningful overall contribution to the downgrade of mortality and morbidity in adult population diagnosed with COVID-19, pregnant women considered to be a part of a high risk group as shown in Table 3 [11]. As statistical data is still insufficient, co-infection with influenza could augment the clinical image of SARS-CoV-2 infection [11].

Another example of seasonal vaccine used for reducing mortality and morbidity in COVID-19 patients is pneumococcal polysaccharide vaccine [11]. Per contra, limited research attempted to determine bacterial co-infections amid SARS-CoV-2 cases, whether if it does not play an important part or the above-mentioned patients benefit from empirical treatment with antimicrobials [11].

TABLE 3. Population at risk of developing

complications from seasonal influenza [10]

\begin{tabular}{l|}
\hline Pregnant women \\
Adults over 65 years old \\
Health care workers \\
Patients with chronic health conditions: \\
- diabetes \\
- cancer \\
- asthma \\
- HIV/AIDS \\
- heart diseases \\
- chronic kidney disease
\end{tabular}

\section{CONCLUSIONS}

Pregnancy is an important period in the life of the future mother, with unique physiological changes. In the context of the new coronavirus, with increasing number of cases all over the world, statistical data and studies on COVID-19 and pregnancy are still limited. Health care providers aim to identify safe and adequate treatment options in pregnant women with SARS-CoV-2 in order to decrease the risk of infection and secondary complications. The role of triage in the prevention and control of SARS-CoV-2 infection is essential, as well as apperception of the main onset symptoms such as high temperature, cough, lethargy, body weakness or gastrointestinal manifestations. Maternal fetal vertical transmission is possible and current recommendations are to isolate a SARS-CoV-2 positive mother from the newborn. As the pandemic is still emerging and in order to safely guide future mothers and breastfeeding women on SARS-CoV-2 infection, additional data and studies are needed.

Taking into account most of the studies it seems to be clear that clear that COVID-19 is less severe in pregnancy than the 2 previous coronavirus infections: severe acute respiratory syndrome-related coronavirus (SARS) and Middle East respiratory syndrome-related coronavirus (MERS). Nevertheless, there are pregnant women that developpe severe forms of SARS-CoV-2 infection and require critical care including mechanical ventilation. Very few pregnant patients and their infants could die as consequence of COVID-19 [4].

Although there is a coherence of most of the study results related to SARS-CoV-2 infection in pregnancy, there are still discrepancies that need more and larger studies in order to clarify debatable aspects.

\section{Acknowledgement}

All authors equally contributed to this article.

Conflict of interest: none declared Financial support: none declared

\section{REFERENCES}

1. Khalil A, Kalafat E, Benlioglu C. SARS-CoV-2 infection in pregnancy: A systematic review and metaanalysis of clinical features and pregnancy outcomes. EClinicalMedicine. 2020 Aug;25:100446.

2. Pastick KA, Nicol MR, Smyth E. A Systematic Review of Treatment and Outcomes of Pregnant Women with COVID-19 - A Call for Clinical Trials. Open Forum Infect Dis. 2020 Aug 13;7(9):ofaa350.

3. Bolton MJ, Weirick ME, Goodwin EC. SARS-CoV-2 seroprevalence among parturient women in Philadelphia. Sci Immunol. $2020 \mathrm{Jul}$ 29;5(49):eabd5709.

4. Delahoy MJ, Whitaker M, O'Halloran A. Characteristics and Maternal and Birth Outcomes of Hospitalized Pregnant Women with Laboratory-Confirmed COVID-19 - COVID-NET, 13 States, March 
1-August 22, 2020. MMWR Morb Mortal Wkly Rep. 2020 Sep 25;69(38):1347-1354.

5. Prabhu M, Cagino K, Matthews KC, et al. Pregnancy and postpartum outcomes in a universally tested population for SARS-CoV-2 in New York City: a prospective cohort study. BJOG. Published online July 7, 2020.

6. Khalil A, von Dadelszen P, Draycott T, Ugwumadu A, O'Brien P, Magee $\mathrm{L}$. Change in the incidence of stillbirth and preterm delivery during the COVID-19 pandemic. JAMA. 2020;324(7):705-706.

7. Ahlberg M, Neovius M, Saltvedt S, et al. Association of SARS-CoV-2 test status and pregnancy outcomes. Research Letter, JAMA. Published online September 23, 2020.
8. Mendoza M, Garcia-Ruiz I, Maiz N, et al. Pre-eclampsia-like syndrome induced by severe COVID-19: a prospective observational study. BJOG. 2020;127(11):1374-1380.

9. Wald ER, Schmit KM, Gusland DY. A Pediatric Infectious Disease Perspective on COVID-19. Clin Infect Dis. 2020 Aug 7;ciaa1095.

10. Juan J, Gil MM, Rong Z, et al. Effect of coronavirus disease 2019 (COVID -19\} on maternal, perinatal and neonatal outcome: systematic review. Ultrasound Obstet Gynecol. 2020 Jul; 56(1):15-27.

11. Thindwa D, Quesada MG, Liu Y. Use of seasonal influenza and pneumococcal polysaccharide vaccines in older adults to reduce COVID-19 mortality, Vaccine. 2020 Jul 22;38(34):5398-5401. 